UFTP-435/1997

Duke-TH-97-140

\title{
Nonequilibrium fluid-dynamics in the early stage of ultrarelativistic heavy-ion collisions
}

\author{
J. Brachmann, A. Dumitru, J.A. Maruhn, H. Stöcker, W. Greiner \\ Institut für Theoretische Physik der J.W. Goethe-Universität \\ Postfach 111932, D-60054 Frankfurt a.M., Germany
}

D.H. Rischke

Department of Physics, Duke University

Durham, NC 27708-0305, U.S.A.

March 1997 


\begin{abstract}
To describe ultrarelativistic heavy-ion collisions we construct a three-fluid hydrodynamical model. In contrast to one-fluid hydrodynamics, it accounts for the finite stopping power of nuclear matter, i.e. for nonequilibrium effects in the early stage of the reaction. Within this model, we study baryon dynamics in the BNL-AGS energy range. For the system $\mathrm{Au}+\mathrm{Au}$ we find that kinetic equilibrium between projectile and target nucleons is established only after a time $t_{C M}^{e q} \approx 5 \mathrm{fm} / \mathrm{c} \simeq 2 R_{A u} / \gamma_{C M}$. Observables which are sensitive to the early stage of the collision (like e.g. nucleon flow) therefore differ considerably from those calculated in the one-fluid model.
\end{abstract}




\section{Introduction}

One of the central interests of heavy-ion physics is the study of the equation of state (EoS) of hot and highly compressed nuclear matter [1]. The success of the hydrodynamical model [2, 3] in describing the gross properties of heavy ion reactions at BEVALAC energies indicated that a collective and equilibrated state of highly compressed nuclear matter is indeed created in collisions of heavy nuclei. In particular, experiments (qualitatively) confirmed [4]

1. enhanced particle emission into preferred solid angles in collisions of a heavy target penetrated by a light projectile with velocity larger than the speed of sound in nuclear matter, indicating the occurrence of a mach cone [5, 6],

2. collective flow of matter in the reaction plane (bounce-off) and perpendicular to the reaction plane (squeeze-out) in semiperipheral collisions of heavy nuclei [6, 7],

3. the linear increase of the pion multiplicity with beam energy due to entropy production. In this model entropy is produced in the compression shock front, the subsequent expansion proceeds via simple waves which - in the ideal fluid limit - conserve entropy and thus the pion multiplicity [8].

These successes motivated the application of the (relativistic) hydrodynamical model to higher-energy heavy-ion reactions. At very high baryon densities and / or temperatures a phase transition from ordinary hadronic matter to a quark-gluon plasma (QGP) is expected [1, 9]. In the (one-fluid) hydrodynamical model the energy and baryon densities necessary for this phase transition are already reached in the BNL-AGS energy regime [10]. Within this model, the effects of a (first order-like) phase transition lead to

1. a plateau in the excitation function of $\left\langle p_{T}\right\rangle$ [11, 
2. a plateau in the excitation function of the pion multiplicity [12],

3. a local minimum in the excitation function of the collective nucleon flow $\left\langle p_{x}^{\text {dir }} / N\right\rangle$ [13, 10],

4. a prolonged lifetime of the system at the "softest point" of the EoS [14].

However, the question arises whether this model is still valid at ultrarelativistic energies (AGS and above), where these effects are predicted. In the one-fluid model instantaneous thermalization of projectile, target, and produced particles is assumed. Therefore, momentum conservation leads to immediate complete stopping at the contact surface (the nuclei do not penetrate each other) and then energy conservation requires that all the kinetic energy is converted into internal excitation of the stopped matter. As a consequence, huge (midrapidity) energy densities, temperatures, and baryon densities are produced. Thus, the conditions for the phase transition are already reached at comparatively low bombarding energy.

In this paper we extend the hydrodynamical model in such a way that thermalization of projectile and target does not happen instantaneously. Within our model, projectile and target decelerate gradually while penetrating each other. We study the influence of these nonequilibrium effects on baryon stopping, directed baryon flow, and the compression of the nuclei. Based on these results, we comment on the validity of the one-fluid hydrodynamical model at ultrarelativistic energies and our expectations concerning the above mentioned predictions of this model. 


\section{The three-fluid model}

In this section we present the physical motivation for introducing three fluids and discuss in detail the interaction sourceterms (see also refs. 115, 16, 17]).

\subsection{Physical motivation}

The description of the early stage of a heavy-ion collision within the framework of ideal one-fluid hydrodynamics tends to become more and more unrealistic, when nuclei collide at AGS-energies $(\sqrt{s} \approx 5 A G e V)$ and above. The major reason for that is that the assumption of instantaneous local thermalization of projectile, target and produced particles fails at ultrarelativistic bombarding energies. This is due to the finite stopping power of nuclear matter. As a consequence, one-fluid hydrodynamics overestimates the baryon and energy densities at midrapidity during the early stage of the reaction. This in turn yields a high pressure in the central region, which produces a large sideward flow in the reaction plane (the so-called bounce-off) [10].

To get a rough estimate of the compression at midrapidity, one may employ the onedimensional shock model [18] with an ultrarelativistic ideal gas $\operatorname{EoS}(p=\epsilon / 3)$ for the shocked matter. The baryon and energy densities are then given by

$$
\begin{aligned}
n / n_{0} & =4 \gamma_{c m}-3 / \gamma_{c m} \\
\epsilon / \epsilon_{0} & =4 \gamma_{c m}^{2}-3
\end{aligned}
$$

$n_{0} \simeq 0.16 \mathrm{fm}^{-3}$ and $\epsilon_{0} \simeq 0.15 \mathrm{GeV} / \mathrm{fm}^{3}$ denote ground state baryon and energy density of nuclear matter. The linear increase of the central baryon density with $\gamma_{c m} \propto \sqrt{s}$ becomes questionable for $\gamma_{c m} \gg 1$, since the assumption of instantaneous thermalization of projectile and target matter ceases to be valid. 
To account for nonequilibrium effects in the early stage of the reaction we introduce several fluids in our model. Considering collisions of single protons at that high energies, one observes a strongly forward-backward-peaked rapidity distribution [19]. Each proton loses about one unit of rapidity and is deflected only by a small angle. The projectile and the target protons remain separated in phase space, if the incident energy is high enough, and can be identified as the projectile respectively target proton even after the collision. This means that to first approximation the two baryon flows (projectile and target) do not exchange baryon charge. For this reason we consider two fluids that correspond to projectile (fluid 1) and target nucleons (fluid 2). Due to inelastic nucleon-nucleon scattering, energy in form of newly produced particles is deposited at midrapidity. Since the two nucleonic fluids do not populate midrapidity in the beginning of the reaction, if the initial rapidity gap was large enough, the newly created particles are also separated in phase space. This motivates to collect them in a third fluid - the fireball.

In our approach projectile and target fluids penetrate each other and decelerate gradually, not instantaneously. Due to inelastic $N N$ scattering the fireball is dynamically produced during the compressional phase of the reaction. Since the time scale of energy deposition is rather short, we neglect the rescattering of the nucleonic fluids with the fireball during this early stage. Later, when all three fluids populate the same phase space region and thermalize, they should be merged into one. Then, hydrodynamical expansion becomes the dominant process. 


\subsection{Multi-fluid relativistic ideal hydrodynamics}

The basic equations of relativistic one-fluid hydrodynamics are the conservation of energy and momentum,

$$
\partial_{\mu} T^{\mu \nu}=0
$$

If we assume an ideal fluid (i.e., neglecting dissipative effects), the energy-momentum tensor $T^{\mu \nu}$ reads [2]

$$
T^{\mu \nu}=(\epsilon+p) u^{\mu} u^{\nu}-p g^{\mu \nu}
$$

where $\epsilon(x)$ is the energy density in the local rest frame, $p(x)$ the pressure, $u^{\mu}(x)=\gamma(1, \vec{v})$ the 4-velocity field (normalized to unity, $u^{\mu} u_{\mu}=1$ ), and $g^{\mu \nu}=\operatorname{diag}(1,-1,-1,-1$ ) the metric tensor.

In addition, there may exist several conserved currents $j_{i}^{\mu}$, for which the continuity equations

$$
\partial_{\mu} j_{i}^{\mu}=0
$$

hold. We will consider only one such conserved current, namely the net baryon current

$$
j^{\mu}=n u^{\mu}
$$

where $n(x)$ denotes the net baryon density in the local rest frame.

As computational frame we choose the frame where the longitudinal velocities of projectile and target nuclei are of equal magnitude. In this frame equations (2, 14) read

$$
\begin{aligned}
\partial_{t} E+\partial_{i}\left(E v^{i}\right) & =-\partial_{i}\left(p v^{i}\right), \\
\partial_{t} M^{j}+\partial_{i}\left(M^{j} v^{i}\right) & =-\partial_{j} p, \\
\partial_{t} R+\partial_{i}\left(R v^{i}\right) & =0 .
\end{aligned}
$$


$E, \vec{M}, R$ denote energy, momentum, and baryon density in the computational frame. They are related to the local rest frame quantities $\epsilon, p$ and $n$ via

$$
\begin{aligned}
E & \equiv T^{00}=\gamma^{2}(\epsilon+p)-p \\
M^{i} & \equiv T^{i 0}=\gamma^{2}(\epsilon+p) v^{i} \\
R & \equiv j^{0}=\gamma n
\end{aligned}
$$

The system (6.7) of coupled differential equations is closed via the assumption of local thermodynamic equilibrium and by specifying an $\operatorname{EoS}$ in the form $p(\epsilon, n)$.

In hydrodynamics for $N$ fluids, we split the energy-momentum tensor and the baryon current of the total system into a sum of $N$ terms,

$$
\begin{aligned}
T^{\mu \nu} & =T_{1}^{\mu \nu}+T_{2}^{\mu \nu}+\cdots+T_{N}^{\mu \nu}, \\
j^{\mu} & =j_{1}^{\mu}+j_{2}^{\mu}+\cdots+j_{N}^{\mu} .
\end{aligned}
$$

The total system is now subdivided into $N$ individual fluids. Note that eqs. (8) hold locally, i.e. different fluids may coexist at the same space-time point. Each individual energymomentum tensor $T_{l}^{\mu \nu}$ and baryon current $j_{l}^{\mu}$ does not need to be conserved, since the $N$ subsystems may exchange energy, momentum and baryon charge:

$$
\begin{aligned}
\partial_{\mu} T_{l}^{\mu \nu} & =F_{l}^{\nu}, \\
\partial_{\mu} j_{l}^{\mu} & =S_{l} \quad(l=1, \cdot, N) .
\end{aligned}
$$

$F_{l}^{\nu}$ denote energy and momentum sources of fluid $l$, and $S_{l}$ the baryon charge exchange for each fluid. Due to eqs. (2, (1), i.e. the conservation of total energy, momentum and baryon charge, we have the additional equations

$$
\begin{aligned}
& F_{1}^{\nu}+F_{2}^{\nu}+\cdots+F_{N}^{\nu}=0 \\
& S_{1}+S_{2}+\cdots+S_{N}=0
\end{aligned}
$$


Following the discussion in section 2.1, we choose $N=3$ and identify fluid 1 with the projectile and fluid 2 with the target nucleons. Fluid 3 collects the energy loss of projectile and target nucleons due to inelastic collisions, and will thus be identified with the produced particles. At time $t=0$ (in the computational frame), the third fluid is not yet existent: $j_{3}^{\mu}(t=0, \vec{x})=0, \quad T_{3}^{\mu \nu}(t=0, \vec{x})=0$.

\subsection{Coupling terms between the fluids}

We now discuss the couplings between the three fluids, based on the assumption that they are well separated in rapidity.

We first discuss the interaction between projectile and target. The total one-particle distribution function can be written as the sum of two individual distribution functions for projectile and target nucleons, respectively:

$$
f(t, \vec{x}, \vec{p})=f_{P}(t, \vec{x}, \vec{p})+f_{T}(t, \vec{x}, \vec{p})
$$

Due to the strongly forward-backward peaked cross section (see discussion in section 2.1) in high energy $p p$-collisions it is possible to identify the projectile respectively target nucleon even after the reaction. The nucleon scattered into the forward hemisphere belongs by definition to the same flow as the one previously heading in this direction. Consequently, projectile and target fluid do not exchange baryon charge: $S_{1}=S_{2}=0$. From eq. (10) and the fact that the fireball is also separated in phase space from the nucleonic fluids, it follows that the fireball remains net baryon free, $S_{3}=0$.

The source terms of the energy-momentum tensor $F_{l}^{\nu}$ in eq. (9) describe the energy respectively momentum loss of fluid $l$ per volume and per unit time. We define

$$
F_{l}^{\nu}=n_{1} n_{2}\left\langle v_{M \varnothing l l e r} \int_{\tilde{p}_{\|}^{\prime}>0} \mathrm{~d} \sigma_{\mathrm{NN} \rightarrow \mathrm{NX}}\left(p^{\prime}-p\right)^{\nu}\right\rangle \quad, \quad \mathrm{d} \sigma_{\mathrm{NN} \rightarrow \mathrm{NX}}=\sigma_{\mathrm{NN} \rightarrow \mathrm{NX}} \frac{\mathrm{d}^{3} p^{\prime}}{E^{\prime}}
$$


This is simply the collision rate per volume $n_{1} n_{2} v_{M ø l l e r} \mathrm{~d} \sigma$ for scattering into the invariant phase space volume $\mathrm{d}^{3} p^{\prime} / E^{\prime}$ times the average 4-momentum loss $\left(p^{\prime}-p\right)^{\nu}$ in a single binary $N N$-collision. $\sigma_{\mathrm{NN} \rightarrow \mathrm{NX}}^{i n v}$ denotes the invariant differential cross section. $p^{\nu}, p^{\nu}$ are the 4momenta of the particle after and before the collision, respectively. The integration extends over the forward hemisphere of the cross section only $\left(\tilde{p}_{\|}^{\prime}>0\right)$. Quantities with a tilde refer to the center-of-mass frame of the binary $N N$ collision. The $n_{i}$ denote the local rest frame baryon densities of projectile and target, as defined in eqs. (5) and (7). $v_{\text {Møller }}$ denotes the invariant relative velocity of the two fluids [20]:

$$
v_{M \varnothing l l e r}=\sqrt{\left(u_{1}^{\mu} u_{2 \mu}\right)^{2}-1} .
$$

The $\langle\cdot\rangle$-averaging over the individual particle distribution functions provides a smearing of the sourceterms (accounting for the thermal and Fermi momenta of the particles), especially of the Møller velocity. It is negligible during the compressional stage of the reaction since the internal velocities within each fluid element are small compared to the Møller velocity of the colliding fluids. We therefore ignore this averaging. Instead, when thermal and relative velocities of the colliding fluids become comparable in the later stage of the collision, the fluids are unified (see section 2.5).

In principle it is always possible to split the sourceterms in a symmetric and an antisymmetric part with respect to the fluid indices $(1 \leftrightarrow 2)$ :

$$
\begin{aligned}
& \partial_{\mu} T_{1}^{\mu \nu}=f_{\text {exchange }}^{\nu}-f_{\text {loss }}^{\nu}, \\
& \partial_{\mu} T_{2}^{\mu \nu}=-f_{\text {exchange }}^{\nu}-f_{\text {loss }}^{\nu}, \\
& \partial_{\mu} T_{3}^{\mu \nu}=2 f_{\text {loss }}^{\nu} .
\end{aligned}
$$

The antisymmetric term $f_{\text {exchange }}^{\nu}$ describes the exchange of energy and momentum between projectile and target fluid, while $f_{\text {loss }}^{\nu}$ denotes the loss of energy and momentum transferred 
to the fireball.

Following [15, 16, 17] we split the integral in eq. (12) into two moments,

$$
\begin{aligned}
& F_{1}^{\nu}=\frac{1}{2} n_{1} n_{2} v_{M ø l l e r}\left[\left(p_{2}-p_{1}\right)^{\nu} \sigma_{P}(s)-\left(p_{2}+p_{1}\right)^{\nu} \sigma_{E}(s)\right], \\
& F_{2}^{\nu}=\frac{1}{2} n_{2} n_{1} v_{M ø l l e r}\left[\left(p_{1}-p_{2}\right)^{\nu} \sigma_{P}(s)-\left(p_{1}+p_{2}\right)^{\nu} \sigma_{E}(s)\right] .
\end{aligned}
$$

Since we neglect the $\langle\cdot\rangle$-averaging, the particle momenta are simply given by $p_{1,2}^{\nu}=m_{N} u_{1,2}^{\nu}$. The moments of the cross section $\sigma_{E}$ and $\sigma_{P}$ are defined as

$$
\sigma_{E}(s)=\int_{\tilde{p}_{\|}^{\prime}>0} \mathrm{~d} \sigma_{\mathrm{NN} \rightarrow \mathrm{NX}}\left(1-\frac{\tilde{E}^{\prime}}{\tilde{E}}\right) \quad, \quad \sigma_{P}(s)=\int_{\tilde{p}_{\| \mid}^{\prime}>0} \mathrm{~d} \sigma_{\mathrm{NN} \rightarrow \mathrm{NX}}\left(1-\frac{\tilde{p}_{\|}^{\prime}}{\tilde{p}_{\|}}\right)
$$

and parametrize the mean energy respectively longitudinal momentum loss in a single nucleonnucleon collision. $E, \vec{p}$ are energy and momentum of the nucleon before the collision, while $E^{\prime}, \overrightarrow{p^{\prime}}$ denote the same quantity after the collision. Again, the integration is carried out only over the forward hemisphere of the cross section $\left(\tilde{p}_{\|}^{\prime}>0\right)$.

Comparing eqs. (14) and eqs. (15) we see that $\sigma_{E}$ corresponds to the symmetric term and leads to the production of the fireball. Analogously, $\sigma_{P}$ is the antisymmetric term describing the exchange of energy and momentum between projectile and target fluids. Also, from this form of the coupling terms it immediately follows that the third fluid is produced at midrapidity: in the CMS $\overrightarrow{p_{1}}=-\overrightarrow{p_{2}}$ and thus $\overrightarrow{F_{3}}=\overrightarrow{0}$. The hydrodynamical eqs. (10) and the specific form of the sourceterms, eq. (15), can also be derived from the Boltzmann equation 17].

We employ the parametrizations of $\sigma_{P, E}$ from the two-fluid model of refs. [15, 16, 17]. There the cross sections of free binary $N N$ collisions $\left(N N \rightarrow N^{*} X\right)$ have been used to evaluate the moments $\sigma_{P, E}$. The incident baryons are assumed to be nucleons, whereas in the final state we sum over all baryons $N^{*}$. The rescattering with the fireball $(N \pi \rightarrow X)$ is not considered, since the contribution to the total cross section outside the $\Delta$-resonance is 
small at high bombarding energies. Since we focus on the dynamics of the nucleons in this paper, we do not expect this to play an important role.

\subsection{Equations of state}

To close the coupled system of differential equations (9) we assume local thermodynamic equilibrium within each fluid (but not in the total system !) and an EoS for each fluid.

For the target and projectile fluids we employ an EoS of an ideal nucleon gas plus compression energy:

$$
p=\zeta\left(\epsilon-E_{c} n\right)+p_{c}
$$

$E_{c}$ is the compression energy per nucleon and $p_{c}$ the compressional pressure. Since the nucleon mass $m_{N}$ is large compared to the typical temperatures of the nucleons, we choose the nonrelativistic limit $\zeta=2 / 3$. For the compression energy, we employ the ansatz

$$
E_{c}=\frac{k_{c}}{18 n n_{0}}\left(n-n_{0}\right)^{2}+m_{N}+W_{0}
$$

i.e., $E_{c}$ increases linearly with the baryon density $n$ for $n \gg n_{0} \approx 0.16 \mathrm{fm}^{-3}$. We assume a ground-state (in-)compressibility $k_{c}=300 \mathrm{MeV}$ and a binding energy of $W_{0}=-16 \mathrm{MeV}$. The compressional pressure $p_{c}$ and the velocity of sound $c_{S}$ at temperature $T=0$ are given by

$$
\begin{aligned}
& p_{c}=-\frac{d E_{c}}{d n^{-1}}=n^{2} \frac{d E_{c}}{d n}=\frac{k_{c}}{18 n_{0}}\left(n^{2}-n_{0}^{2}\right), \\
& c_{S}^{2}=\frac{d p}{d \epsilon}=\frac{n / n_{0}}{n / n_{0}-1+9\left(m_{N}+W_{0}\right) / k_{c}} .
\end{aligned}
$$

Ansatz (18) thus ensures $c_{S}(n, T=0)<1$ as long as $k_{c}<9\left(m_{N}+W_{0}\right) \approx 8.3 \mathrm{GeV}$.

For the third fluid we employ an EoS of an ideal gas of $\pi, \eta, \rho, \omega$ mesons with a phase transition to QGP at $T_{c}=160 \mathrm{MeV}$ [21]. However, here we do not discuss the dynamics of the third fluid. 


\subsection{One-fluid transition}

In the later stage of the collision the nucleonic fluids stop. Their relative velocity (Møller velocity) is then comparable to the internal thermal velocities. The two fluids are no longer separated in phase space, so that the main assumption for a two-fluid region does not hold anymore. Moreover, the coupling sourceterms, eqs. (15), cease to be valid, since they do not account for thermal smearing and vanish linearly with the Møller velocity. Thus, two infinitesimal fluid elements with equal fluid velocities, $u_{1}^{\mu}=u_{2}^{\mu}$, would not exchange energy and momentum, even if their temperatures were different. Because the particle velocities are thermally distributed around the fluid velocity, there should be a non-zero coupling that further drives the two fluids into common local thermal equilibrium. Since we do not account for this, the two fluids are merged, if the Møller velocity (13) is comparable to the root-mean-square velocity in a nonrelativistic degenerate Fermi gas or a nonrelativistic Boltzmann gas:

$$
\begin{aligned}
(\Delta v)_{\text {Boltzmann }}^{2} & =\max _{l=1,2}\left\{3 T_{l} / m_{N}\right\}, \\
(\Delta v)_{\text {Fermi }}^{2} & =\max _{l=1,2}\left\{2 \epsilon_{F}\left(n_{l}\right) / m_{N}\right\},
\end{aligned}
$$

where

$$
\epsilon_{F}\left(n_{l}\right)=\left(\frac{3}{2} \pi^{2} n_{l}\right)^{2 / 3} / 2 m_{N}
$$

denotes the Fermi energy. In contrast to [22, 3, 15], the transition is done abruptly without a smooth transition from two to one fluid.

Under the constraining assumption that both fluids flow with a common velocity $\vec{v}$ after unification, the set of eqs. (7) is solved for the local rest frame densities $\epsilon$ and $n$ by inserting the total densities $E=E_{1}+E_{2}, R=R_{1}+R_{2}, \vec{M}=\vec{M}_{1}+\vec{M}_{2}$. By virtue of the EoS, the total pressure $p=p(\epsilon, n)$ is also known. Additive quantities, i.e. the pressure $p=p_{1}+p_{2}$, 
the baryon density $n=n_{1}+n_{2}$ and the energy density $\epsilon=\epsilon_{1}+\epsilon_{2}$ are then redistributed to the individual fluids according to the ratio of the baryon densities in the computational frame $[22,3]$ :

$$
p_{i}=p \frac{R_{i}}{R_{1}+R_{2}} \quad, \quad n_{i}=n \frac{R_{i}}{R_{1}+R_{2}} \quad, \quad \epsilon_{i}=\epsilon \frac{R_{i}}{R_{1}+R_{2}} \quad, \quad i \in\{1,2\} \quad .
$$

Since this procedure changes the individual fluid velocities $v_{i}$ to the common velocity $v$, the propagated quantities $R_{i}, E_{i}, \vec{M}_{i}$ have to be redetermined from $n_{i}, \epsilon_{i}$ and $p_{i}$ according to equations (17) with the common velocity $v$ and the corresponding $\gamma$ factor. Let $\tilde{R}_{i}, \tilde{E}_{i}, \overrightarrow{\tilde{M}}_{i}$ be the resulting quantities. Our special choice of the redistribution, eq. (23), implies that the individual baryon densities in the computational frame are not changed: $\tilde{R}_{i}=R_{i}$.

It can be easily proven that the total quantities $\tilde{E}_{1}+\tilde{E}_{2}=E=E_{1}+E_{2}, \tilde{R}_{1}+\tilde{R}_{2}=$ $R=R_{1}+R_{2}, \tilde{\vec{M}}_{1}+\overrightarrow{\tilde{M}}_{2}=\vec{M}=\vec{M}_{1}+\vec{M}_{2}$ fulfill the one-fluid hydrodynamical eqs. (6). That makes it possible to propagate the fluids separately. Even if that has no physical meaning for the individual fluid, one can tell what amounts in phase space originated from projectile or target, respectively.

Moreover we will use this feature to simulate a one-fluid model with our three-fluid model, by switching off the production of the fireball (setting $\sigma_{E}=0$ ) and forcing the local unification of the two nucleonic fluids at any instant. This is what we will call the onefluid limit of the three-fluid model. Formally, the one-fluid limit corresponds to $\Delta v=\infty$ in eqs. (20,21).

In the present stage of model development, only the one-fluid transition of the nucleonic fluids is implemented. The unification of all three fluids is recommended and in preparation because much of the nucleonic fluids finally end up at midrapidity, where the third fluid is produced. This would slightly increase the pressure in the central region and enhance the directed and radial flow of the nucleons. 


\subsection{Technical realization of the model}

The hydrodynamical eqs. (6) are solved on an Eulerian grid using the SHASTA (SHarp And Smooth Transport Algorithm) [23, 24, 25]. This algorithm was extensively tested in [26, 27]. Each of the three fluids is propagated on its own grid with eight different densities $(R, \vec{M}, E, n, \epsilon, p)$. For each fluid the three-dimensional propagation is performed using the method of time-step splitting (operator splitting) [26], i.e. the 3-divergence operators on both sides of eqs. (6) are not summed over the contracting index $i$. One solves sequentially equations of the form:

$$
\partial_{t} U+\partial_{i}\left(U v_{i}+f\right)=0 \quad, \quad \text { for } i=x, y, z \quad .
$$

Here $U$ stands for either $R, M_{i}$, or $E$ and $-\partial_{i} f$ denotes the corresponding right-hand side of eqs. (6), e.g. in case of $U=E$ and $i=x$ we have $-\partial_{x} f=-\partial_{x}\left(p v_{x}\right)$. The $z$ (beam)-direction is propagated first, those $z$-propagated densities are then propagated in $x$-direction (impact parameter) and finally propagated in $y$-direction (out-of-plane). This order is kept fixed for reasons of simplicity. By permutation the symmetry of the propagation could be enhanced [26]. Obviously the propagational sourceterms $-\partial_{i} f$ are included in the propagation.

In contrast, we exclude in our calculations the coupling sourceterms (right-hand side of eqs. (9)) from the propagation. In our implementation the fluids are first propagated without coupling terms and their local rest frame densities are calculated according to eqs. (7). From these densities the coupling sourceterms (eqs. (15)) are calculated and added. Since this coupling changes the energy and momentum density of the individual fluids, eqs. (7) have to be solved again in order to accomplish the full time step. Applying this procedure it is easy to control the effect of the coupling, e.g. a cell cannot become more than empty by extracting energy from it. In such a case the coupling would not be applied to any of the 
fluids.

In order to minimize the Lorentz contraction we choose the equal-velocity frame as the computational frame. In the equal-velocity frame both projectile and target nucleus have the same velocity (with opposite sign). In the case of two nuclei of the same mass this is identical to the center-of-mass frame. Because of the occurrence of acausalities one should not use a different grid spacing in different spatial directions [28]. Therefore, we use an equidistant grid with grid spacing $\Delta x=\Delta y=\Delta z=0.15 \mathrm{fm}$. This choice was found sufficient to provide convergence in the rapidity distributions. It ensures the correct number of $N N$ collisions with the appropriate colliding energy $\sqrt{s}$. Our time step is $\Delta t=0.4 \Delta x$ to fulfill the Courant-Friedrichs-Lewy criterion [29] for the SHASTA.

As a consequence of eqs. (7) the cell velocity is:

$$
v=\frac{|\vec{M}|}{E+p}
$$

We call a cell acausal if $E<|\vec{M}|$. This happens mainly because in the final step of the SHASTA the propagated density is distributed uniformly over a cell so that, on a too coarse grid, some part of the fluid is transported due to this smoothing procedure and not by real motion. Another possible origin is that due to the coupling of the different fluids too much energy is extracted from a fluid compared to the extracted momentum. We are correcting those acausal cells by rescaling the energy density $E$ with the momentum density:

$$
E_{\text {corrected }}=\left(1+10^{-8}\right) \cdot|\vec{M}|
$$

This corresponds to setting the corrected cells to a maximum velocity $v_{\max }$. By removing the acausal cells instead of correcting them as described above, we found for the $\Delta x=0.15 \mathrm{fm}$ grid used here that less than $5.0 \%$ of the initial baryon charge and $2.0 \%$ of the initial total energy was lost. 


\section{Previous models}

Before presenting results we briefly review some earlier work on which our model is based. The main difference to these approaches is that in none of the predecessor models the produced particles were collected in a third fluid and propagated hydrodynamically. They were two-fluid models only (i.e., $N=2$ in the above equations).

To our knowledge, the first application of multi-fluid hydrodynamics to heavy-ion collisions was discussed in ref. [22]. Similar to our model, fluids 1 and 2 corresponded to the projectile and target nucleons, respectively. They could exchange energy and momentum but no baryons. The couplings between the two fluids were already derived using the underlying concept of binary nucleon-nucleon interactions. However, only elastic nucleon-nucleon scattering (i.e., no particle production) was taken into account. In this model $F_{1}^{\mu}+F_{2}^{\mu}=0$ and thus the sum of $T_{1}^{\mu \nu}$ and $T_{2}^{\mu \nu}$ was conserved.

In ref. [3] a similar model with a more refined coupling (more appropriate for the upper BEVALAC energy range, $E_{L a b}^{K i n} \simeq 2 A G e V$ ) was presented. However, also in this model no midrapidity fluid was considered, i.e. $F_{1}^{\mu}+F_{2}^{\mu}=0$.

In contrast, the authors of [30] investigated the case $F_{1}^{\mu}+F_{2}^{\mu} \neq 0$. Thus, the possibility of inelastic reactions in collisions between projectile and target nucleons was accounted for. However, the antisymmetric (with respect to exchange of the fluid indices $1 \leftrightarrow 2$ ) term in their couplings (diffusion term),

$$
F_{1}^{\mu}-F_{2}^{\mu}=-2 D n_{1} n_{2}\left(u_{1}^{\mu}-u_{2}^{\mu}\right)
$$

involved a diffusion constant $D$, whereas we employ a $\sqrt{s}$-dependent $\sigma_{P}$, cf. eq. (15). Moreover, in their model the symmetric term (drain term) was

$$
F_{1}^{\mu}+F_{2}^{\mu}=-2 q\left(n_{1} u_{2}^{\mu}+n_{2} u_{1}^{\mu}\right)
$$


again with a $\sqrt{s}$-independent $q$. As already mentioned above, the radiative energy losses of fluids 1 and 2 were not collected in a third fluid and propagated hydrodynamically, in contrast to our model. Furthermore, the fluids were propagated in $(1+1)$-dimensions only, thus calculations of the transverse flow of the nucleons were not possible. Finally, since merging of the two fluids into one fluid (when they reach thermal equilibrium) was also not considered, the expansion of the stopped and excited projectile and target fluids and collective flow of spectator matter could not be investigated.

In the two-fluid model of [15, 16, 17], the sourceterms were calculated using experimental data for the elastic and inelastic $N N$ cross sections and do not contain free parameters like $D$ and $q$ in eqs. (27) and (28). There was no energy loss to the fireball below the pion production threshold and the energy deposition in the fireball increases with the c.m. energy $\sqrt{s}$ of the colliding nucleonic fluids. This $\sqrt{s}$ dependence of the sourceterms accounts for the multiple production of particles, mainly pions. However, the authors of [15, 16, 17] considered only two fluids: the energy loss due to the creation of the fireball was accounted for in the propagation of projectile/target fluids, but the fireball itself was not propagated hydrodynamically. In [31] the opposite point of view was taken by studying the production and hydrodynamic expansion of the third fluid in $S+S$ at $200 \mathrm{AGeV}$ (assuming some global interaction between the nucleons of fluids one and two), however, disregarding the compression and heating (and thus the hydrodynamic evolution) of the projectile and target fluids.

In contrast, our three-fluid model propagates all three fluids hydrodynamically and treats the interactions locally in space-time. It thus allows to study spectra of produced particles like thermal pions or photons [21] as well as the dynamics of the nucleons. In this paper we focus on the latter and thus neglect the scattering of the fluid of produced particles with the 
projectile and target fluids. A more refined investigation of various particle spectra, as in [32, is in preparation.

\section{Results}

In this section we present results on compression of projectile respectively target, baryon stopping and flow. We emphasize the deviations from the one-fluid limit which is achieved by setting $\sigma_{E} \equiv 0$ (i.e. no energy loss due to particle production) and by merging the projectile and target fluids at each space-time point from the very beginning.

A remark about the freeze-out should be added here. Usually a hydrodynamical treatment becomes unrealistic when reaching some local freeze-out criterion, i.e. for example the local temperature drops below the freeze-out temperature [33] or the local density becomes smaller than a certain freeze-out density. Fluid elements would then freeze-out locally (e.g. in boostinvariant hydrodynamics [34] according to their eigentime). While such a local freezeout criterion would be a more realistic scenario, it is very difficult to realize, especially in a fully three-dimensional calculation.

Due to these difficulties and in order not to introduce additional free parameters (like the freeze-out temperature and / or density), we prefer to discuss only fluid instead of particle distributions. Our results (e.g. the $\mathrm{dN} / \mathrm{dY}$-spectra, cf. section 4.2) are therefore compared to the one-fluid limit, not directly to experimental data. Also due to this reason, we show the time evolution of the observables under consideration at fixed center-of-mass time (not at fixed proper time). 


\subsection{Density evolution}

In section 2.1 we claimed that in a one-fluid model much higher densities are reached than in multi-fluid models. Figure 1 shows the average baryon density of the projectile nucleus as a function of time. The averaging is defined as

$$
\left\langle n_{P}\right\rangle=\frac{\int \mathrm{d}^{3} x R_{P}(x) n_{P}(x)}{\int \mathrm{d}^{3} x R_{P}(x)}
$$

where $R_{P}$ denotes the projectile baryon density in the computational frame, cf. section 2.2. Thus it is a measure for the compression of the projectile only. In the one-fluid limit the

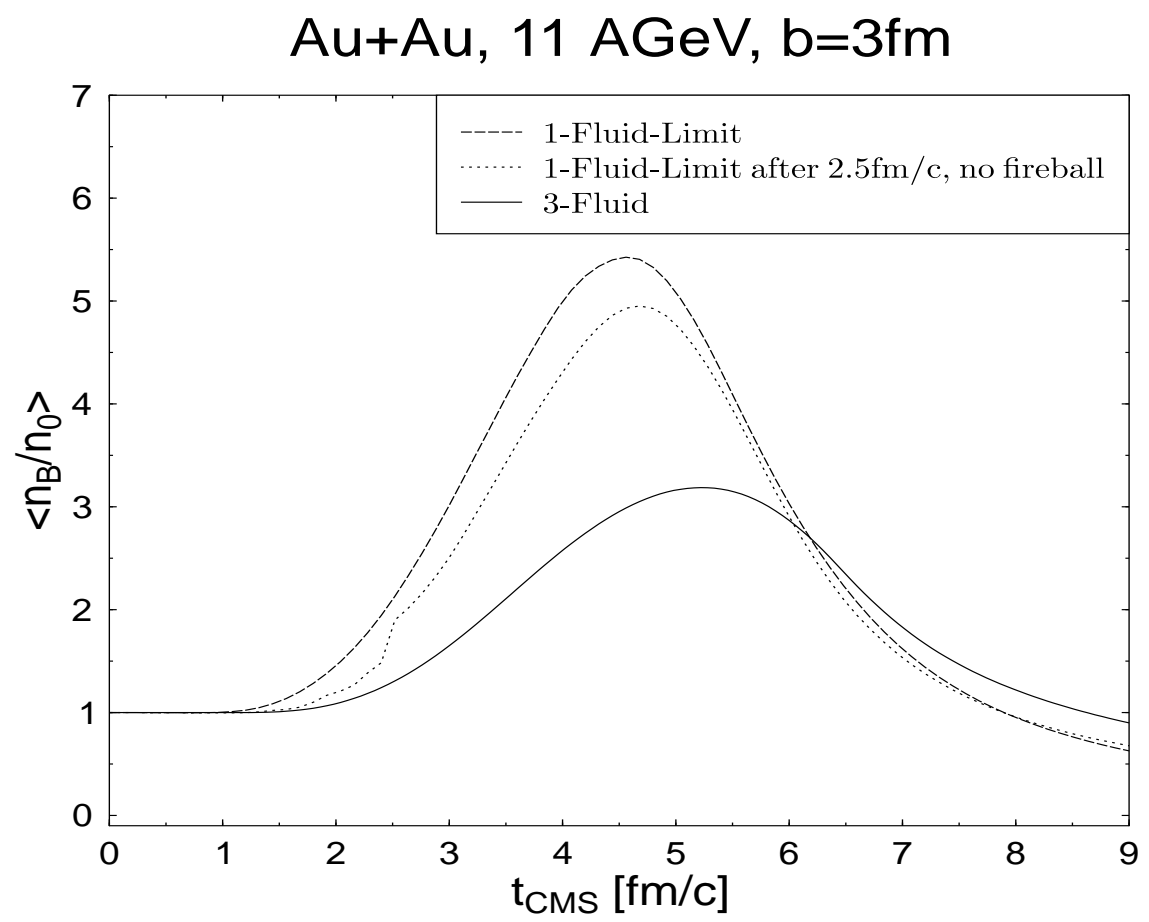

Figure 1: Average baryon density of the projectile.

maximum projectile density is almost a factor of two higher and is reached earlier than in the three-fluid model.

The dotted line shows a calculation without fireball production $\left(\sigma_{E}=0\right)$, like in the one-fluid 
limit, but the unification was performed after $2.5 \mathrm{fm} / \mathrm{c}$. The evolution first follows more or less the three-fluid curve but yields slightly higher densities. It was already pointed out in [30 that neglecting the energy loss due to particle production results in higher excitations in multi-fluid models. At $2.5 \mathrm{fm} / \mathrm{c}$, when instantaneous unification like in the one-fluid limit is applied, the curve jumps towards the one-fluid limit but does not reach the full height since the nuclei already penetrated partially.

The reason for the lower compression of the baryons in the three-fluid model is the finite stopping length of nucleons in nuclear matter. This is illustrated in Fig. 2 which shows the energy density profile of the projectile fluid along the beam axis. In the one-fluid limit the shock wave which moves outwards can be clearly seen, leaving behind compressed matter with energy density $\epsilon \approx 20 \epsilon_{0}$. Also, no flow through the plane $z=0$ exists (corresponding to instantaneous, complete stopping of the projectile). We checked that the shock-wave velocity and the energy density of the shocked matter in this three-dimensional calculation agrees satisfactorily well with the one-dimensional shock model. In contrast, in the three-fluid model there is no discontinuity in the baryon or energy density profile which separates the central region from the incoming ground-state nuclear matter. Due to their non-vanishing mean-free path some projectile nucleons do pass the $(z=0)$-plane and the shock front is broadened considerably. As a consequence, the maximum energy density of the projectile matter does not exceed $10 \epsilon_{0}$.

\subsection{Baryon stopping}

Figures 3 and 1 compare the time evolution of the rapidity distributions of the nucleonic fluids 1,2 in the one-fluid limit and the three-fluid model, respectively. We again stress that both figures show fluid distributions, i.e. the particle momenta are not thermally smeared. 


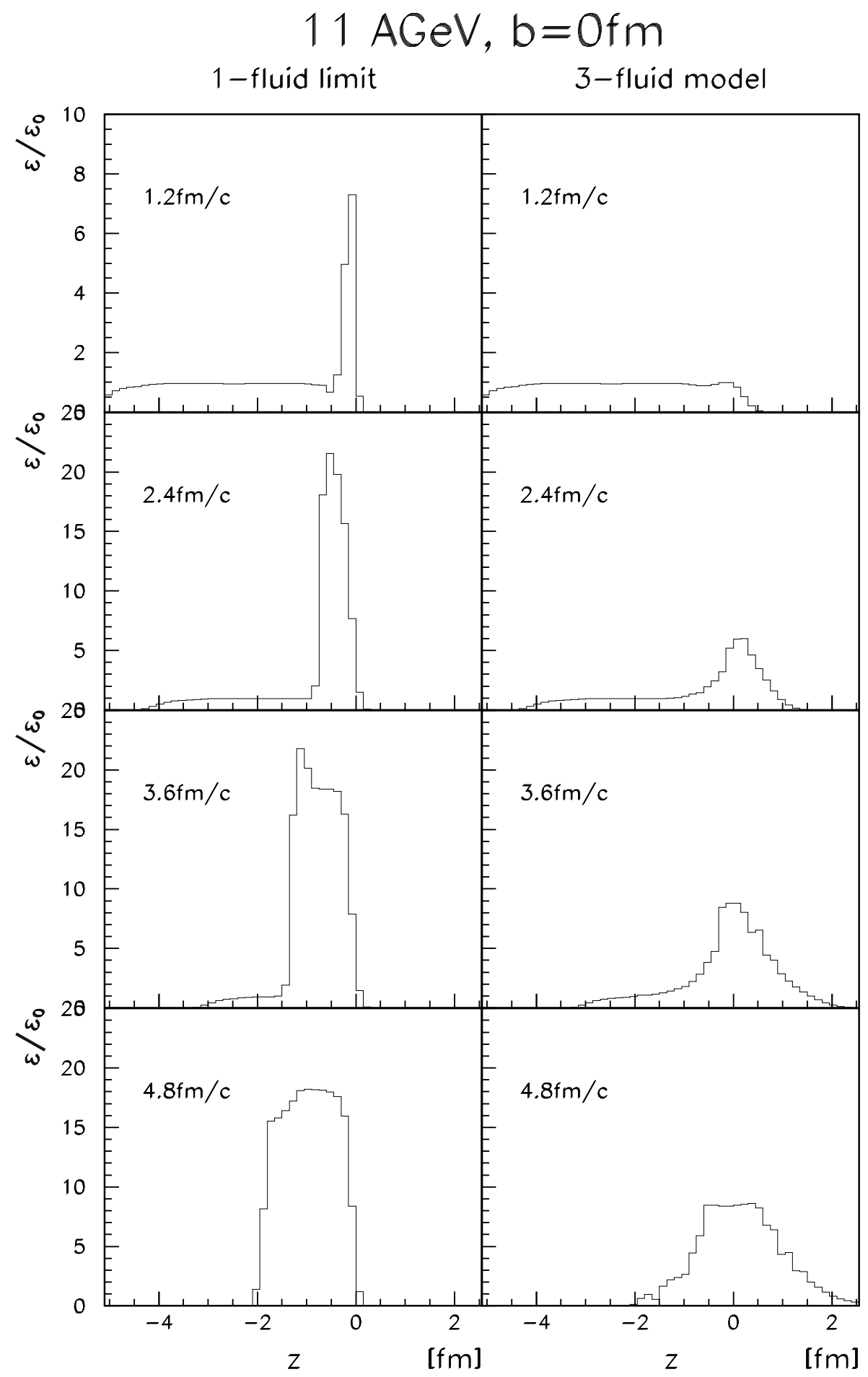

Figure 2: Energy density profile (in units of $\epsilon_{0} \approx 0.15 \mathrm{GeV} / \mathrm{fm}^{3}$ ) of the projectile fluid along the beam axis (in the plane $x=y=0$ ). 
The time steps in the two figures are not equal, since the compression reaches its maximum faster in the one-fluid limit, cf. Fig. 11.

The major difference between one- and multi-fluid models as claimed in section 2.1 is becoming obvious: At $1.2 \mathrm{fm} / \mathrm{c}$ the fluid rapidity distribution in the one-fluid limit (Fig. 3 , upper left panel) shows clearly that participant matter stops immediately at midrapidity when the nuclei touch, with nothing in between midrapidity and the projectile or target region, respectively. Comparing the rapidity distributions at $4.8 \mathrm{fm} / \mathrm{c}$ (Fig. 3), middle right panel, and Fig. 4, upper right panel), in the one-fluid limit the colliding baryons are piling up at midrapidity while in the three-fluid model they are shifting smoothly towards midrapidity. Nevertheless, looking at the later stages, i.e. $6.0 \mathrm{fm} / \mathrm{c}$, even in the three-fluid model the colliding nuclei become more or less stopped within about one unit in rapidity before reaccelarating. Thus, the coupling sourceterms, parametrized by free binary nucleon-nucleon collisions, are strong enough to yield a sufficient stopping.

A benefit of our unification procedure is that we can track the individual nucleonic fluids even after unification since they are formally propagated on separate grids. We observe that projectile and target are changing position in the $\mathrm{dN} / \mathrm{dY}$ in the one-fluid limit, while they keep the sign in rapidity in the three-fluid model, i.e., that projectile and target are reflected in the one-fluid limit, while heading on in their initial direction in the three-fluid model.

In the late stage of the reaction expansion is becoming the most important process which is reaccelerating the fluids again in both the one-fluid limit and the three-fluid model.

\subsection{Equilibration}

For an equilibrated system the pressure is isotropic. In contrast, while equilibration is not yet established, the transverse and longitudinal pressures (as defined in kinetic theory 35) will 


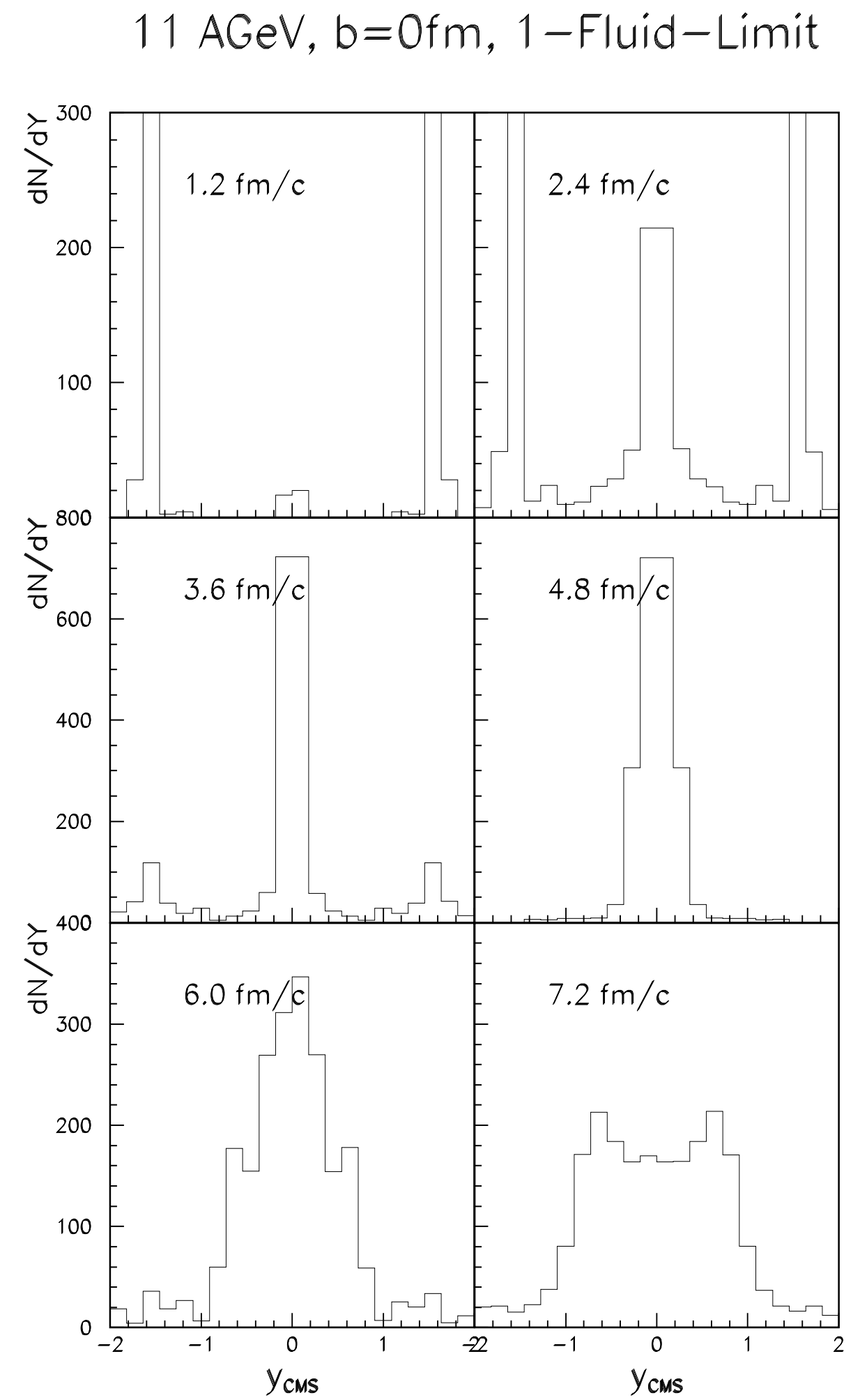

Figure 3: The evolution of the baryon fluid $\mathrm{dN} / \mathrm{dY}$ in the one-fluid limit. 


\section{AGeV, b=0fm, 3-Fluid-Model}

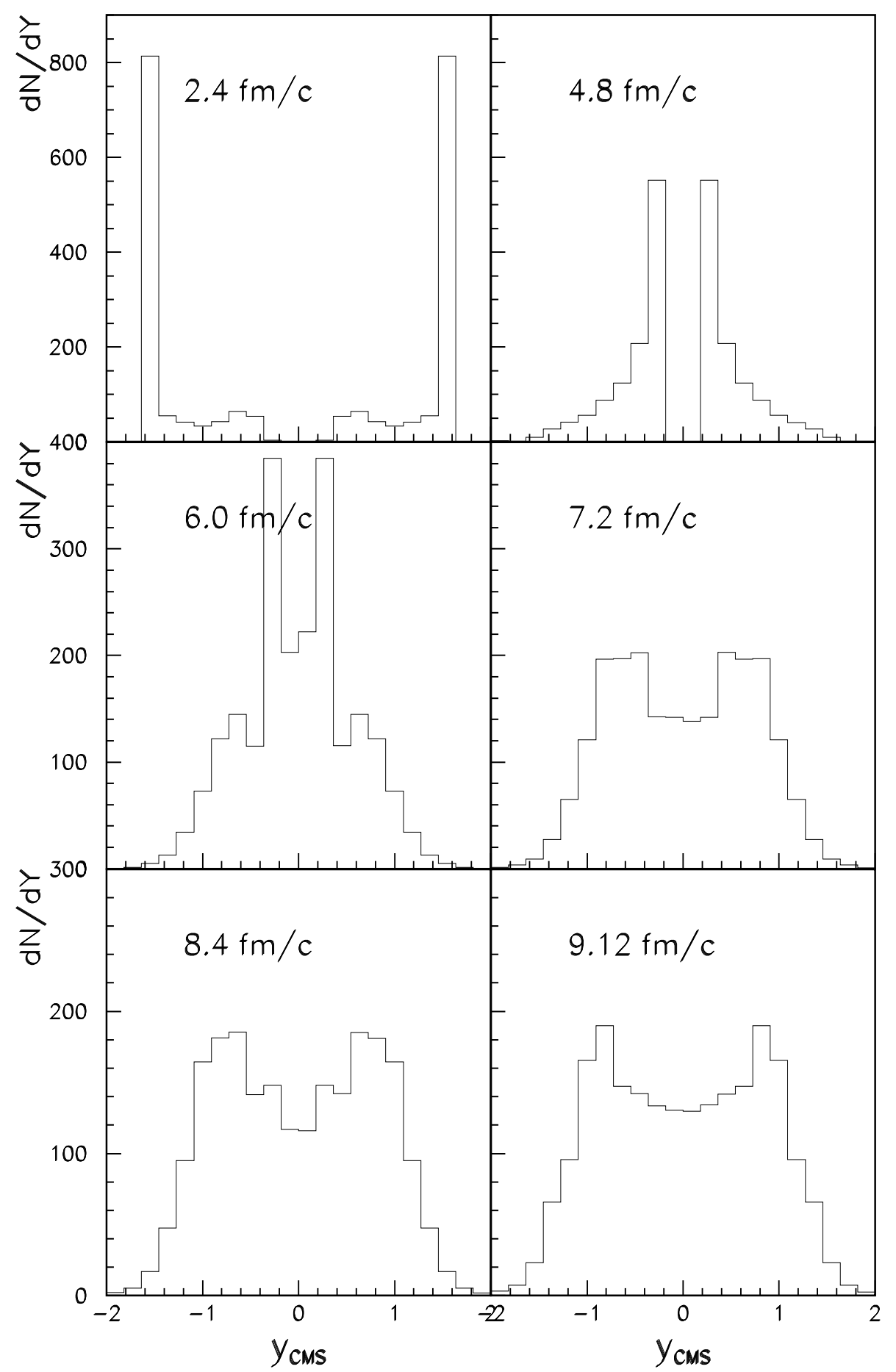

Figure 4: The evolution of the baryon fluid dN/dY in the three-fluid model. 
differ from each other. In our approach, this reflects in a deviation of the sum of the partial pressures (of projectile and target fluids) from the equilibrium pressure. This is investigated in Fig. 5 by calculating the ratio $p_{1}+p_{2}$ to $p_{\text {equil }}$ that a unification of the nonequilibrated fluids would yield . The same analysis is done for the rest frame baryon densities,

$$
\left\langle\frac{p_{1}+p_{2}}{p_{\text {equil }}}\right\rangle=\frac{\int \mathrm{d}^{3} x \frac{p_{1}(x)+p_{2}(x)}{p_{\text {equil }}(x)}}{\int \mathrm{d}^{3} x} \quad, \quad\left\langle\frac{n_{1}+n_{2}}{n_{\text {equil }}}\right\rangle=\frac{\int \mathrm{d}^{3} x \frac{n_{1}(x)+n_{2}(x)}{n_{\text {equil }}(x)}}{\int \mathrm{d}^{3} x} .
$$

The volume integration runs in one case only over a thin central layer perpendicular to the beam axis, so that most of the matter traverses this plane during the reaction and in the second case over the whole overlap region of fluids 1 and 2 . The ratio is by definition equal to unity in the case of unified fluids, for in that case $p_{1}$ and $p_{2}$ just denote the partial pressures. One observes that kinetic equilibrium is established only after a time $t_{C M}^{e q} \approx(5-6) \mathrm{fm} / \mathrm{c} \simeq$ $2 R_{A u} / \gamma_{C M}$. A similar equilibration time scale was also extracted from a cascade model [36]. Thus, observables which are sensitive to this early stage of the reaction [37 (as e.g. the nucleon flow discussed in the next section) will deviate considerably from their values in the one-fluid limit. One also sees (cf. dashed curve) that the merging of projectile and target nucleons starts at $t_{C M} \approx 5 \mathrm{fm} / \mathrm{c}$, when the collision terms $F_{1,2}^{\mu}$ have almost established kinetic equilibrium.

It seems that the matter in the central plane equilibrates faster than the whole overlap region. However one has to recall that matter is passing through this thin layer, so that the ratios evaluated at different times refer to different parts of matter. Moreover, the respective matter corresponds to matter trailing the fronts of the nuclei. On the other hand, for the overlap region more or less the same matter remains within the volume of integration at all times.

\footnotetext{
${ }^{1} p_{\text {equil }}$ and $n_{\text {equil }}$ are calculated from eqs. (7) by inserting $E=E_{1}+E_{2}, \vec{M}=\vec{M}_{1}+\vec{M}_{2}$ and $R=R_{1}+R_{2}$ for the left-hand side.
} 


\section{Au-Au, $11 \mathrm{AGeV}, \mathrm{b}=0$}
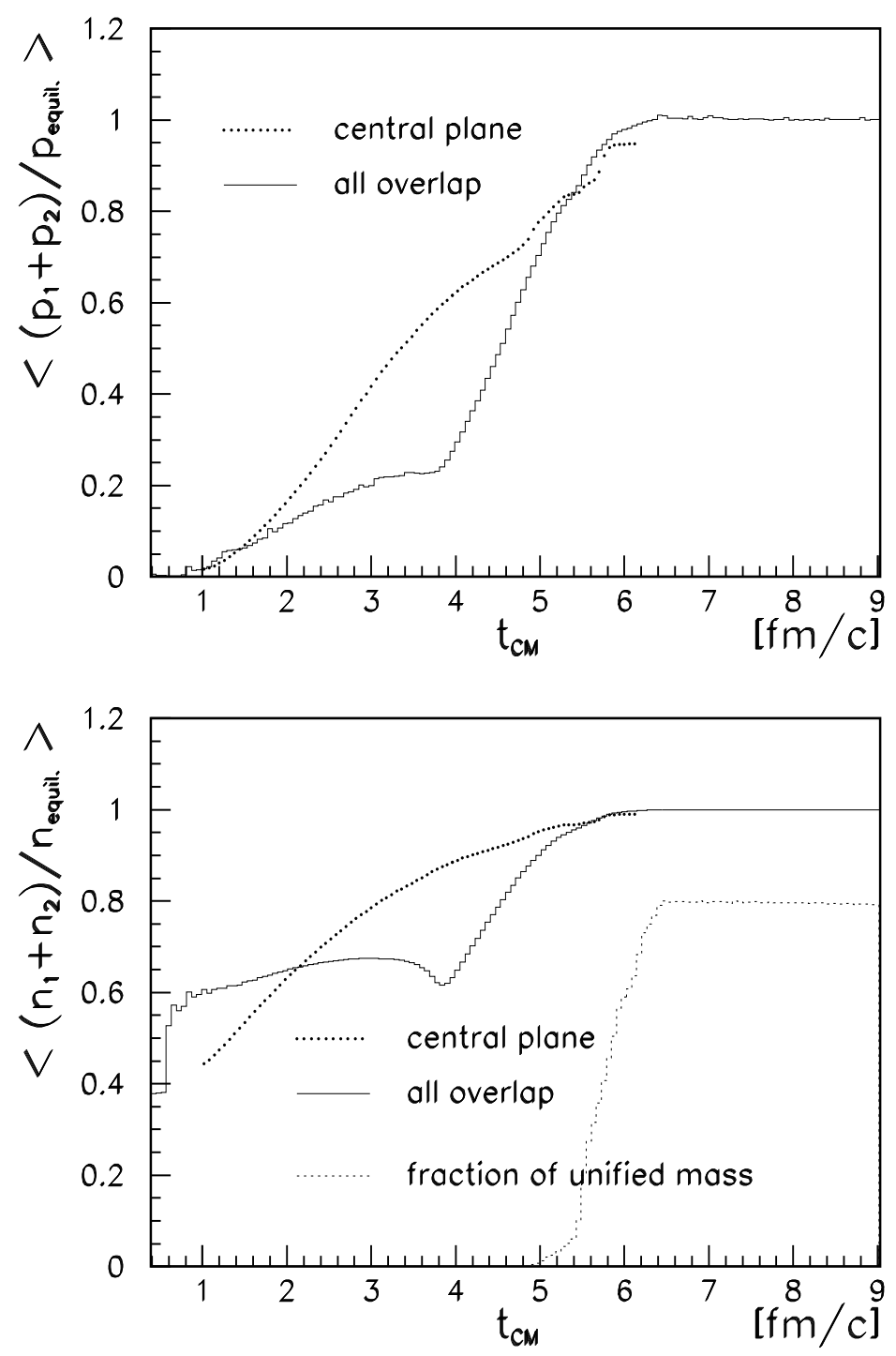

Figure 5: Evolution of sum of nonequilibrium pressures and rest frame baryon densities compared to the corresponding equilibrium values (ratios). 


\subsection{Nucleon flow}

As a measure for the in-plane transverse flow we investigate the mean transverse momentum per nucleon $\left\langle p_{x} / N\right\rangle$ as a function of rapidity $y$. We define $\left\langle p_{x} / N\right\rangle$ as follows:

$$
\left\langle p_{x} / N\right\rangle(y)=\frac{\int_{y} \mathrm{~d}^{3} x R(\vec{x}) m_{N} u_{x}(\vec{x})}{\int_{y} \mathrm{~d}^{3} x R(\vec{x})}
$$

Here $u_{x}=\gamma v_{x}$ denotes the component of the local 4 -velocity field in the direction of the impact parameter and $m_{N}$ the nucleon rest mass. We assume $m_{N} u_{x}$ to be a measure for the component of the nucleonic momentum in $x$-direction without thermal smearing. The volume integration is performed over all fluid elements (projectile and target) around a given rapidity $y$. Figures 6 and 0 show the time evolution of the $\left\langle p_{x} / N\right\rangle$-distributions for one-fluid limit and three-fluid model, respectively.

During the reaction pressure builds up and "spectator" matter is deflected. Therefore, $\left\langle p_{x} / N\right\rangle$ is increasing. The one-fluid limit shows higher maximum $\left\langle p_{x} / N\right\rangle$ than the three-fluid model, i.e. produces more sideward flow, as claimed in section 2.1. In the later stages the flow is still $\approx 280 \mathrm{MeV} / \mathrm{c}$ in the one-fluid limit, which is higher than the final value of the three-fluid model of $\approx 120 \mathrm{MeV} / \mathrm{c}$. The flow for the three-fluid model does not change any more from about $6.0 \mathrm{fm} / \mathrm{c}$ on and thus is a reasonable measure for the final amount of flow after freeze-out.

In [10] an excitation function of the directed flow $\left\langle p_{x}^{d i r}\right\rangle$ has been investigated for a hadron gas EoS in comparison to an EoS with a phase transition to QGP. This one-fluid model predicted a minimum of the directed flow $\left\langle p_{x}^{d i r}\right\rangle$ around the phase transition region. $\left\langle p_{x}^{d i r}\right\rangle$ was defined as [10]:

$$
\left\langle p_{x}^{d i r}\right\rangle=\frac{1}{N} \int \mathrm{d} y \frac{\mathrm{d} N}{\mathrm{~d} y} \frac{y}{|y|}\left(\left\langle p_{x} / N\right\rangle(y)\right)
$$

This is basically the weighted mean of the function $\left\langle p_{x} / N\right\rangle(y)$, eq. (31). 
$\mathrm{Au}+\mathrm{Au}(11 \mathrm{AGeV}), \mathrm{b}=3 \mathrm{fm}$, 1-Fluid-Limit

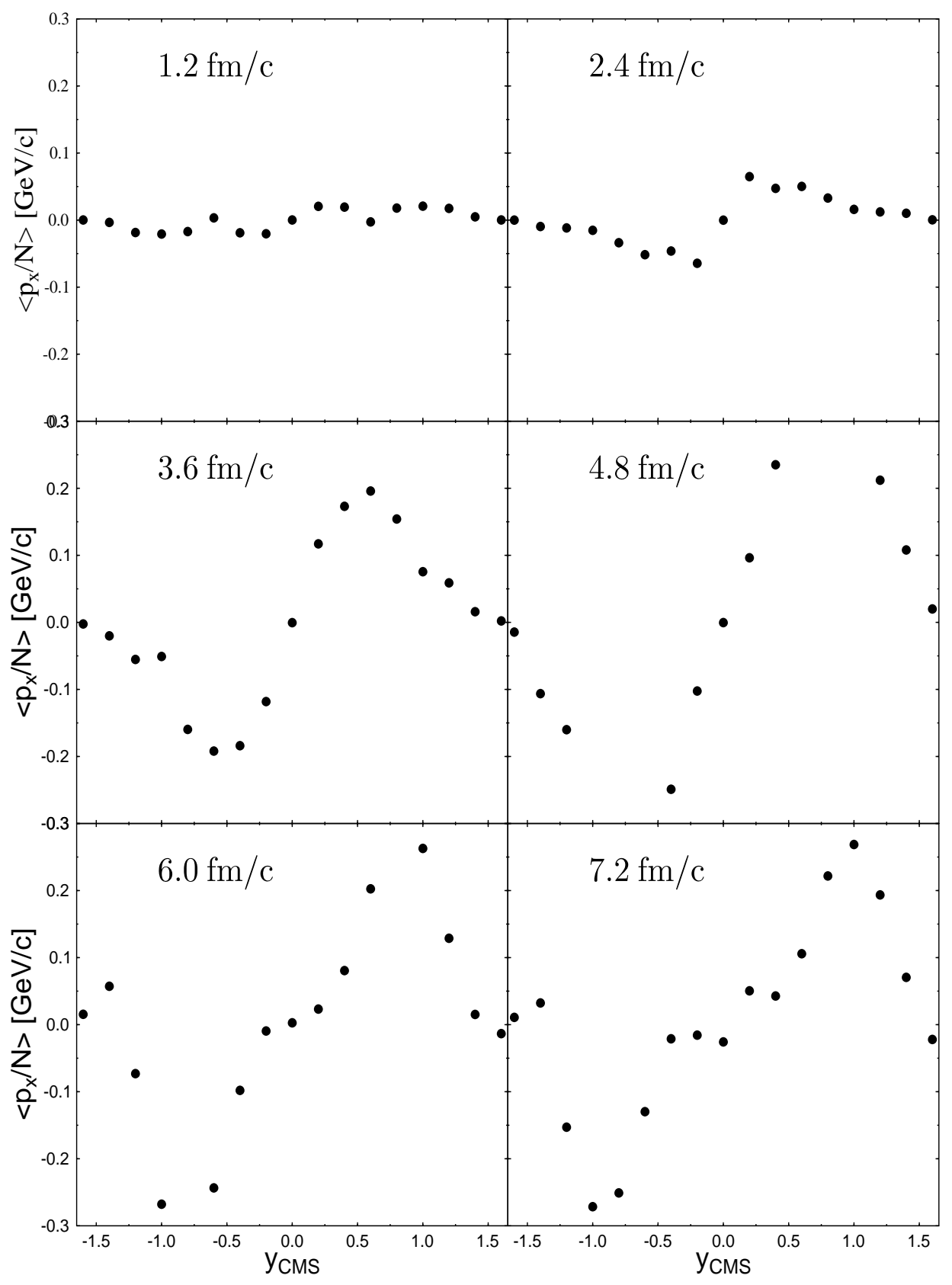

Figure 6: The evolution of $\left\langle p_{x} / N\right\rangle$ in the one-fluid limit. 


\section{$\mathrm{Au}+\mathrm{Au}(11 \mathrm{AGeV}), \mathrm{b}=3 \mathrm{fm}, 3$-Fluid-Model}

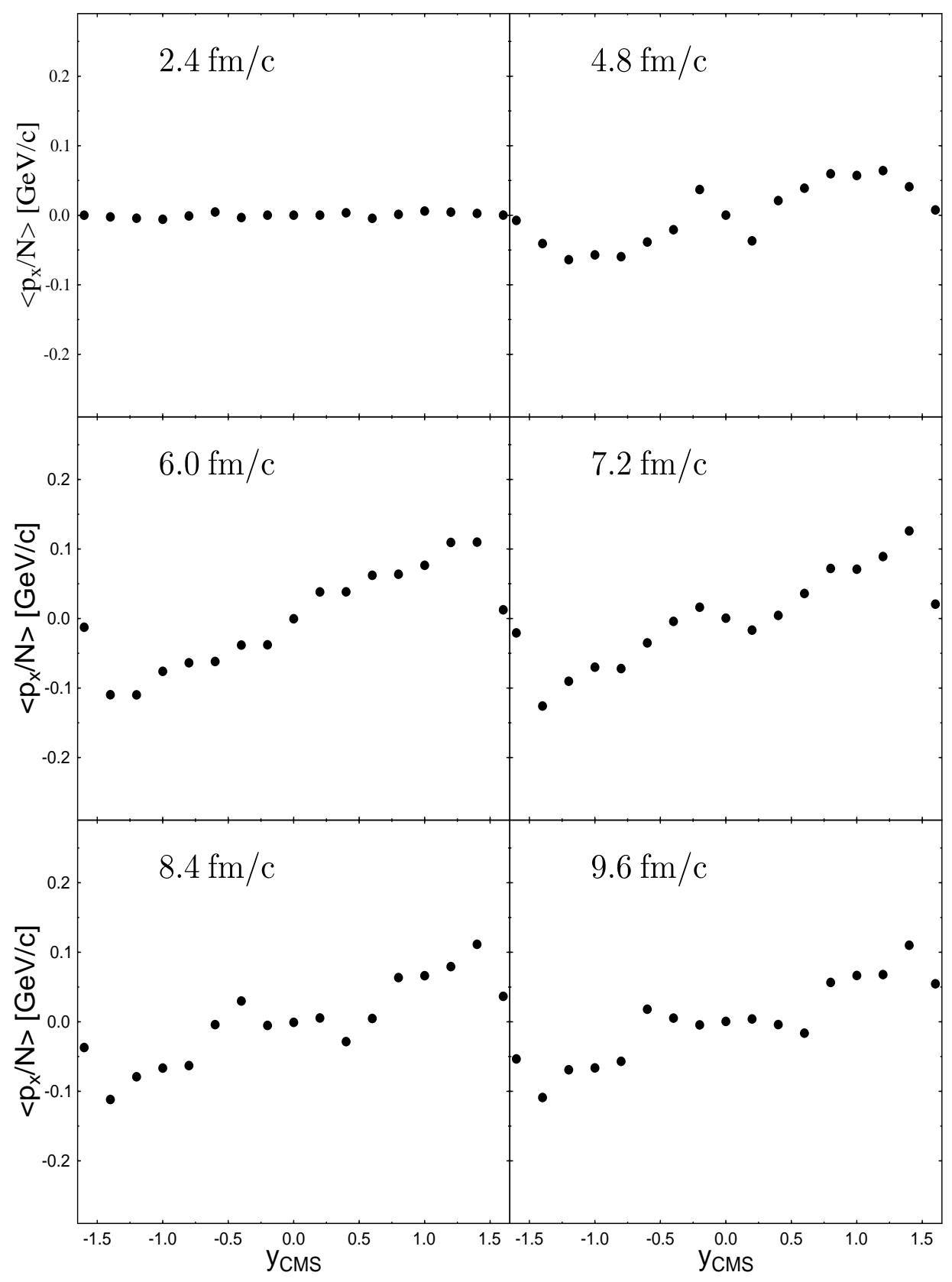

Figure 7: The evolution of $\left\langle p_{x} / N\right\rangle$ in the three-fluid model. 
For our EoS (17), $\left\langle p_{x}^{d i r}\right\rangle=120 \mathrm{MeV} / \mathrm{c}$ in the one-fluid limit, whereas $\left\langle p_{x}^{d i r}\right\rangle=40 \mathrm{MeV} / \mathrm{c}$ in the three-fluid model. Even with our crude EoS without a phase transition (17) the directed nucleon flow is as low as in the one-fluid model with a phase transition to QGP [10]. Obviously, the effect of the nonequilibrium effects is of the same order of magnitude as the softening of the EoS due to a (first order) phase transition. Some implications of kinetic nonequilibrium in the early stage of ultrarelativistic heavy-ion collisions were already sketched in [37].

\section{Summary, conclusion and outlook}

In this paper we presented a three-fluid hydrodynamical model which allows to account for nonequilibrium effects between target, projectile, and produced particles during the early stage of the collision. We discussed that due to the nonvanishing thermalization time scale, this model yields a

1. lower energy density and temperature,

2. lower pressure,

3. less baryonic compression,

4. and a different velocity distribution

of the nucleons at early times as compared to the one-fluid hydrodynamical model (which assumes instantaneous local thermalization between projectile, target, and produced particles). As a consequence, we expect that the

1. directed nucleon flow, 
2. entropy production (pion multiplicity),

3. thermal electromagnetic radiation [21,

4. lifetime of the hot and dense central region

differ considerably in the three-fluid as compared to the one-fluid model. These results suggest that the predictions of the one-fluid model (as summarized in the introduction) have to be modified by taking nonequilibrium effects into account.

We especially point out that in three-fluid model the projectile and target nucleons reach kinetic equilibrium only after $t_{C M}^{e q} \approx 2 R / \gamma_{C M}$. Thus the directed nucleon flow (emerging at early times) is smaller and in particular much less sensitive to the EoS as compared to the one-fluid limit. This is due to the fact that the flow of the nucleons of fluid $i$ is caused mainly by the partial pressure $p_{i}$ and not by the full $p_{e q}$ (see Fig. 5).

In the future more realistic equations of state for the projectile and target fluids (incorporating a phase transition to a quark-gluon plasma at high energy densities) have to be implemented and the excitation functions for the pion multiplicity, the mean transverse momentum, and the directed nucleon flow have to be reinvestigated.

Acknowledgements: We gratefully acknowledge helpful discussions with L. Satarov and I. Mishustin. The work of D.H.R. has been supported by the Director, Office of Energy Research, Division of Nuclear Physics of the Office of High Energy and Nuclear Physics of the U.S. Department of Energy under contract no. DE-FG-02-96ER-40945. 


\section{References}

[1] H. Stöcker, W. Greiner: Phys. Rep. 137 (1986) 277

[2] L.D. Landau, E.M. Lifshitz: "Fluid Mechanics", Pergamon Press, 1959

[3] R.B. Clare, D. Strottman: Phys. Rep. 141 (1986) 177

[4] R. Stock et al.: Phys. Rev. Lett. 49 (1982) 1236;

H.A. Gustafsson et al.: Phys. Rev. Lett. 52 (1984) 1590;

R.E. Renford et al.: Phys. Rev. Lett. 53 (1984) 763;

J. Harris et al.: Phys. Lett. B153 (1985) 377;

H.G. Ritter et al.: Nucl. Phys. A447 (1985) 3c;

K.G.R. Doss et al.: Phys. Rev. Lett. 57 (1986) 302;

R. Stock: Phys. Rep. 135 (1986) 259;

J. Harris et al.: Phys. Rev. Lett. 58 (1987) 463;

H.H. Gutbrod, A.M. Postkanzer, H.G. Ritter: Rep. Prog. Phys. 52 (1989) 1267;

H.H. Gutbrod et al.: Phys. Lett. B216 (1989) 267; Phys. Rev. C42 (1990) 640 ;

for a topical review see K.-H. Kampert: J. Phys. G15 (1989) 691

[5] W. Scheid, R. Ligensa, W. Greiner: Phys. Rev. Lett. 21 (1968) 1479;

W. Scheid, W. Greiner: Z. Phys. 226 (1969) 364;

W. Scheid, H. Müller, W. Greiner: Phys. Rev. Lett. 32 (1974) 741

[6] H. Stöcker, J.A. Maruhn, W. Greiner: Phys. Rev. Lett. 44 (1980) 725

[7] G.F. Chapline, M.H. Johnson, E. Teller, M.S. Weiss; Phys. Rev. D8 (1973) 4302;

G. Buchwald, G. Gräbner, J. Theis, J.A. Maruhn, W. Greiner, H. Stöcker: Phys. Rev. Lett. $52(1984) 1594$ 
[8] H. Stöcker, W. Greiner, W. Scheid: Z. Phys. A286 (1978) 121

[9] see, e.g.,

E.V. Shuryak: Phys. Lett. B78 (1978) 150;

B. Müller: "The Physics of the Quark-Gluon Plasma", Lecture Notes in Physics 225, Springer, 1985;

L. McLerran: Rev. Mod. Phys. 58 (1986) 1021

[10] D.H. Rischke, Y. Pürsün, J.A. Maruhn, H. Stöcker, W. Greiner: Heavy Ion Phys. 1 (1995) 309

[11] L. van Hove: Phys. Lett. B118 (1982) 138

[12] K.A. Bugaev, M.I. Gorenstein, D.H. Rischke: Phys. Lett. B255 (1991) 18

[13] M. Gyulassy, W. Greiner: Ann. Phys. 109 (1977) 485;

L. Bravina, L.P. Csernai, P. Levai, D. Strottmann: Phys. Rev. C50 (1994) 2161;

L. Bravina, N. Amelin, L.P. Csernai, P. Levai, D. Strottmann: Nucl. Phys. A566 (1994) $461 \mathrm{c}$

[14] S. Pratt: Phys. Rev. D33 (1986) 1314;

G. Bertsch: Nucl. Phys. A498 (1989) 173e;

S. Pratt: Phys. Rev. C49 (1994) 2722;

C.M. Hung, E.V. Shuryak: Phys. Rev. Lett. 75 (1995) 4003;

D.H. Rischke, S. Bernard, J.A. Maruhn: Nucl. Phys. A595 (1995) 346;

D.H. Rischke, M. Gyulassy: Nucl. Phys. A608 (1996) 479

[15] I.N. Mishustin, V.N. Russkikh, L.M. Satarov: Sov. J. Nucl. Phys. 48 (1988) 454 
[16] I.N. Mishustin, V.N. Russkikh, L.M. Satarov: Nucl. Phys. A494 (1989) 595;

I.N. Mishustin, L.M. Satarov, V.N. Russkikh: in "Relativistic Heavy Ion Physics" (eds. D. Strottman and L.P. Csernai), vol. 1, World Scientific (Singapore), 1991, p.179 and Sov. J. Nucl. Phus. 54 (1991) 459

[17] L.M. Satarov: Sov. J. Nucl. Phys. 52 (1990) 264

[18] A.M. Taub: Phys. Rev. 74 (1948) 328

[19] V. Blobel et al.: Nucl. Phys. B69 (1974) 454

[20] C. Møller: K. Dan. Vidensk. Selsk. Mat. Fys. Medd. 23 (1945) 1

[21] A. Dumitru, U. Katscher, J.A. Maruhn, H. Stöcker, W. Greiner, D.H. Rischke: Phys. Rev. C51 (1995) 2166; Z. Phys. A 353 (1995) 187

[22] A.A. Amsden, A.S. Goldhaber, F.H. Harlow, J.R. Nix: Phys. Rev. C17 (1978) 2080

[23] J.P. Boris, D.L. Book: J. Comput. Phys. 11 (1973) 38

[24] D.L. Book, J.P. Boris, K. Hain: J. Comput. Phys. 18 (1975) 248

[25] J.P. Boris, D.L. Book: J. Comput. Phys. 20 (1976) 397

[26] D.H. Rischke, S. Bernard, J.A. Maruhn: Nucl. Phys. A595 (1995) 346

[27] D.H. Rischke, Y. Pürsün, J.A. Maruhn: Nucl. Phys. A595 (1995) 383

[28] B. Waldhauser, D.H. Rischke, U. Katscher, J.A. Maruhn, H. Stöcker, W. Greiner: Z. Phys. C54 (1992) 459

[29] R. Courant, K. Friedrichs, H. Lewy: Math. Ann. 100 (1928) 32 
[30] H.W. Barz, B. Kämpfer: Phys. Lett. B206 (1988) 399

[31] U. Katscher, D.H. Rischke, J.A. Maruhn, W. Greiner, I.N. Mishustin, L.M. Satarov: Z. Phys. A346 (1993) 209

[32] P. Braun-Munzinger, J. Stachel, J.P. Wessels, N. Xu: Phys. Lett. B344 (1995) 43; Phys. Lett. B365 (1996) 1

[33] see, e.g.

I.N. Mishustin, L.M. Satarov: Sov. J. Nucl. Phys. 37 (1983) 532;

U. Ornik, F.W. Pottag, R.M. Weiner: Phys. Rev. Lett. 63 (1989) 2641;

F.S. Navarra, M.C. Nemeth, U. Ornik, S. Paika: Phys. Rev. C45 (1992) R2552;

E. Schnedermann, U. Heinz: Phys. Rev. C47 (1993) 1738;

D.K. Srivastava, J. Alam, S. Chakrabarty, B. Sinha, S. Raha: Ann. Phys. (N.Y.) 228 (1993) 104;

B.R. Schlei, U. Ornik, M. Plümer, D. Strottman, R.M. Weiner: Phys. Lett. B376 (1996) 212 ;

S. Bernard, J.A. Maruhn, W. Greiner, D.H. Rischke: Nucl. Phys. A605 (1996) 566

[34] J.D. Bjorken: Phys. Rev. D27 (1983) 140

[35] S.R. de Groot, W.A. van Leeuwen, Ch.G. van Weert: "Relativistic Kinetic Theory", Noth Holland, Amsterdam, 1980

[36] J. Brachmann, A. Dumitru, C. Spieles, J.A. Maruhn, H. Stöcker, W. Greiner: contribution to the GSI annual scientific report 1997 (in print) / nucl-th 9701045

[37] H. Sorge: Preprint SUNY-NTG 96-40 / nucl-th 9610026;

Preprint SUNY-NTG 97-1 / nucl-th 9701012 